\title{
Homogenization of Dirichlet Problems with Convex Bounded Constraints on the Gradient
}

\author{
T. Champion and L. De Pascale
}

\begin{abstract}
We give a simpler proof of a conjecture of A. Bensoussan, J. L. Lions and G. Papanicolaou on the homogenization of Dirichlet problems with convex bounded constraints on the gradient. Our proof is based on a regularization technique for the constrained functionals as well as on a fine approximation technique.
\end{abstract}

Keywords: Homogenization, Moreau-Yosida approximations, Convex constraints on the gradients

AMS subject classification: 74099, 49N20, 41A99

\section{Introduction}

In this paper we study the asymptotic behaviour as the positive parameter $\varepsilon$ goes to 0 of the sequences of solutions of the problems

$\left(\mathbf{P}_{\varepsilon}\right) \min \left\{F_{\varepsilon}(v)+\int_{\Omega} \beta(x) v(x) d x: v \in \mathcal{C}_{0}(\Omega)\right\}$

where $\mathcal{C}_{0}(\Omega)$ denotes the set of continuous functions on $\bar{\Omega}$ which vanish on the boundary $\partial \Omega$ and the functionals $F_{\varepsilon}$ are given on $\mathcal{C}_{0}(\Omega)$ by

$$
F_{\varepsilon}(v)= \begin{cases}\int_{\Omega} f\left(\frac{x}{\varepsilon}, D v(x)\right) d x & \text { if } v \in W^{1, \infty}(\Omega) \cap \mathcal{C}_{0}(\Omega) \\ +\infty & \text { elsewhere }\end{cases}
$$

and where $\Omega$ is an open bounded subset of $\mathbb{R}^{N}$ with $|\partial \Omega|=0, \beta \in L^{1}(\Omega)$ and $f$ is a function satisfying the following conditions:

T. Champion: Lab. d'Anal. Non Lin. Appl., U.F.R. des Sc. et Techn., Univ. de Toulon et du Var, Av. de l'Université, BP 132, F-83957 La Garde Cedex, France; champion@univ-tln.fr

L. De Pascale: Dip. di Mat. Appl., Univ. di Pisa, Via Bonanno Pisano 25/B, IT-56126 Pisa, Italy; depascal@dm.unipi.it

ISSN 0232-2064 / \$2.50 C Heldermann Verlag Berlin 
(1) $(x, \xi) \mapsto f(x, \xi) \in[0,+\infty]$ is measurable in $(x, \xi),(0,1)^{N}$-periodic in $x$, lower-semicontinuous and convex in $\xi$.

(2) $f(\cdot, 0)$ belongs to $L^{1}\left((0,1)^{N}\right)$.

(3) There exists $R>0$ such that $0 \in \operatorname{dom} f(x, \cdot) \subset B(0, R)$ for a.e. $x \in \Omega$.

In the following, we denote by $C(x)=\operatorname{dom} f(x, \cdot)$ the pointwise constraint set. By Hypotheses (1) and (3), $C(x)$ is closed, convex and included in $B(0, R)$ for almost every $x$ in $\Omega$. Notice that $F_{\varepsilon}(v)<+\infty$ implies $D v(x) \in C\left(\frac{x}{\varepsilon}\right)$ a.e., thus $\left(P_{\varepsilon}\right)$ is a family of problems with convex bounded constraints on the gradient. This kind of problem arises in various physical models as for the dielectric breakdown, polycrystal plasticity, or torsional creep problems. We refer to [4, 20 - 22] for a presentation of the applications. Moreover, as explained in [4], this question is also related to the homogenization of variational inequalities.

In 1978, it was conjectured in [4: p. 207 - 214] that if for each $\varepsilon>$ 0 the function $u_{\varepsilon}$ is a minimizer of the corresponding problem $\left(\mathrm{P}_{\varepsilon}\right)$, then the convergent subsequences of the family $\left(u_{\varepsilon}\right)_{\varepsilon}$ converge (in $\mathcal{C}_{0}(\Omega)$ endowed with the topology of uniform convergence) to minimizers of the homogenized problem

$$
\left(\mathbf{P}_{\text {hom }}\right) \min \left\{F_{\text {hom }}(v)+\int_{\Omega} \beta(x) v(x) d x: v \in \mathcal{C}_{0}(\Omega)\right\}
$$

where the homogenized functional $F_{\text {hom }}$ is given on $C_{0}(\Omega)$ by

$$
F_{\text {hom }}(v)= \begin{cases}\int_{\Omega} f_{\text {hom }}(D v(x)) d x & \text { if finite and } v \in W^{1, \infty}(\Omega) \cap \mathcal{C}_{0}(\Omega) \\ +\infty & \text { elsewhere }\end{cases}
$$

while the convex integrand $f_{\text {hom }}$ is defined on $\mathbb{R}^{N}$ by the formula

$$
\begin{aligned}
& f_{\text {hom }}(\xi)= \\
& \inf \left\{\int_{(0,1)^{N}} f(y, \xi+D v(y)) d y: v \in W_{\#}^{1, \infty}\left((0,1)^{N}\right) \cap \mathcal{C}\left((0,1)^{N}\right)\right\} .
\end{aligned}
$$

In the above formula, $W_{\#}^{1, p}\left((0,1)^{N}\right)$ denotes the set of functions of $W_{l o c}^{1, p}\left(\mathbb{R}^{N}\right)$ which are $(0,1)^{N}$-periodic. Our setting is slightly more general than the setting of [4] since the constraint sets $C(x)$ do not need to be balls or even to be balanced.

Here we give a short and self-contained proof of the previous homogenization formula in the general case which fits in the scheme of the proof of [1: p. 106 - 112/Theorem 1.49] (see also [2]). Our proof of the $\Gamma$-convergence of the functionals $\left(F_{\varepsilon}\right)_{\varepsilon}$ to $F_{\text {hom }}$ (see Section 2 for details) thus splits in two independent parts: the estimate for the $\Gamma$-liminf is obtained through the regularization of the functionals $F_{\varepsilon}$ while the $\Gamma$-limsup estimate is obtained through a 
suitable piecewise affine approximation of functions subject to constraints on the gradient. The regularization for the functionals $F_{\varepsilon}$ consists in approximating the integrands of these integral functionals $F_{\varepsilon}$ via inf-convolution with the function $|\cdot|^{N+1}$. For every $\varepsilon>0$, this gives a non-decreasing family $\left(F_{\varepsilon, \lambda}\right)_{\lambda}$ of functionals to which the classical homogenization theory in the Sobolev space $W^{1, N+1}$ apply. The $\Gamma$-lim inf estimate then follows easily from the nondecreasing property. On the other hand, the $\Gamma$-lim sup estimate is obtained through a more technical and involved approximation argument: the main step here is to approximate a given Lipschitz continuous function whose gradient is subject to bounded convex constraints by a family of continuous and piecewise affine functions whose gradients satisfy the same constraints. This can be done under one of the two following additional assumptions on $f$ and $C$ :

$$
\begin{aligned}
& \text { either a) } 0 \text { belongs to the interior of } C_{\text {hom }}=\operatorname{dom}\left(f_{\text {hom }}\right) \\
& \text { or } \quad \text { b) }\left\{\begin{array}{l}
\exists w \in W^{1, \infty}\left((0,1)^{N}\right) \cap \mathcal{C}_{0}\left((0,1)^{N}\right): \\
f_{\text {hom }}(0)=\int_{(0,1)^{N}} f(y, D w(y)) d y .
\end{array}\right.
\end{aligned}
$$

As we shall see, this hypothesis is used to obtain an upper bound for $\Gamma$ $\lim \sup F_{\varepsilon}\left(u^{0}\right)$, where $u^{0}$ is the function which is identically 0 on $\Omega$ (see Lemma 4.6). Part b) of hypothesis (1.1) seems rather technical and in fact reduces to the following statement: the infimum in the definition of $f_{\text {hom }}(0)$ is attained in $W^{1, \infty}\left((0,1)^{N}\right) \cap \mathcal{C}_{0}\left((0,1)^{N}\right)$. This hypothesis is obviously satisfied for example when, for all $x$,

$$
f(x, 0)=\min \{f(x, \xi): \xi \in C(x)\}
$$

in which case $w=0$ fits for part b) of hypothesis (1.1). This property is for example satisfied in the physical models discussed in [21] where $f(x, \xi)=|\xi|^{2}$. Moreover, property (1.2) is always satisfied when $f(x, 0)=0$ and this last condition can be interpreted in continuum mechanics as a consequence of the fact that the parts of a body which do not undergo any deformation do not give contribution to the stored energy.

As this topic is already widely present in the literature (without being exhaustive, we refer to $[6-13,15-16,19,21])$, we think it is important to notice once more that the results proved in this paper are partly known. Our proof does not make use of more or less involved concept (e.g. inner regularization of functionals, dependency on the set) needed in the classical proofs. We also allow as constraints very general convex sets which can degenerate or be very anti-symetric. We also remark that an approach similar to that of the present paper was proposed in $[1,3,19]$, and was also worked out in the case of Neumann problems in [10]. Our contribution consists in a technical simplification of the approach and in an extension of the results to more general domains. In fact, the approximating functionals we choose for the $\Gamma$-lim inf 
are extremely natural and do not require special lemmas. For the $\Gamma$-limsup we use a step by step reconstruction of the limit functional on different classes of functions. However, the way we do it is considerably simple thanks to the approximation used in Lemma 4.10 and to the classes of functions we choose. In particular, the method of calculus of the $\Gamma$-limsup permits to extend the results to non-convex domains $\Omega$ and to avoid various notions of regularization of set functions as the "inner regularization" which is commonly used in this topic.

The plan of the paper is the following: in Section 2 we introduce some notations, we recall the definition and the main properties of the $\Gamma$-convergence and of the inf-convolution of convex functions. Then we introduce the regularized functionals $F_{\varepsilon, \lambda}$ and recall the standard homogenization theory which can be applied to these functionals. The main results are stated and commented in Section 3 while the proof of the $\Gamma$-convergence result is given in Section 4 .

\section{Notations and preliminary results}

2.1 $\Gamma$-convergence. We now recall the notion of $\Gamma$-convergence which will be widely employed in the following. For more details on this tool we refer to the book [18]. Letting $X$ be a metric space, we say that a sequence $\left(F_{n}\right)_{n}$ of functionals from $X$ to $\overline{\mathbb{R}} \Gamma$-converges to $F$ at $x$ if

$$
\Gamma-\liminf _{n \rightarrow+\infty} F_{n}(x)=\Gamma-\limsup _{n \rightarrow+\infty} F_{n}(x)
$$

where $\Gamma$-liminf and $\Gamma$-limsup are defined by

$$
\begin{aligned}
\Gamma-\liminf F_{n}(x) & =\inf \left\{\liminf _{n \rightarrow+\infty} F_{n}\left(x_{n}\right): x_{n} \rightarrow x \text { in } X\right\} \\
\Gamma-\lim \sup F_{n}(x) & =\inf \left\{\limsup _{n \rightarrow+\infty} F_{n}\left(x_{n}\right): x_{n} \rightarrow x \text { in } X\right\} .
\end{aligned}
$$

Moreover, the sequence $\left(F_{n}\right)_{n} \Gamma$-converges to $F$ if it $\Gamma$-converges at every $x$ in $X$. Then the family $\left(F_{\varepsilon}\right)_{\varepsilon>0} \Gamma$-converges to $F$ if $\left(F_{\varepsilon_{n}}\right)_{n \in \mathbb{N}} \Gamma$-converges to $F$ for any sequence $\left(\varepsilon_{n}\right)_{n}$ of positive real numbers converging to 0 .

The following theorem reports fundamental properties of $\Gamma$-convergence (we refer to the first chapters in [18]).

Theorem 2.1. Assume that the sequence $\left(F_{n}\right)_{n} \Gamma$-converges to $F$. Then $F$ is lower semicontinuous on $X$. Moreover, if the family $\left(F_{n}\right)_{n}$ is equicoercive on $X$, then $F$ is coercive too and for any sequence of positive numbers $\varepsilon_{n}$ converging to 0 the following holds:

(1) The sequence $\left(\inf _{X} F_{n}\right)_{n}$ converges to the minimum of $F$ on $X$. 
(2) If $x_{n}$ are such that $F_{n}\left(x_{n}\right) \leq \inf _{X} F_{n}+\varepsilon_{n}$ and $x_{n_{k}}$ converges to $x$ for some subsequence $\left(x_{n_{k}}\right)_{k}$ of $\left(x_{n}\right)_{n}$, then $F(x)=\min _{X} F$.

Remark. The sequence $\left(F_{n}\right)_{n}$ is equicoercive on $X$ if for every $t \in \mathbb{R}$ there exists a compact subset $K$ of $X$ such that $\left\{F_{n} \leq t\right\} \subset K$ for every $n \in \mathbb{N}$.

The following proposition which links the $\Gamma$-convergence with the pointwise convergence in a monotone case will be useful. We denote by $\bar{F}$ the lower semi-continuous envelope (or relaxed functional) of $F$ on $X$.

Proposition 2.2. If $F_{n}$ is a non-decreasing sequence on $X$, then

$$
\Gamma-\lim _{n \rightarrow+\infty} F_{n}=\lim _{n \rightarrow \infty} \bar{F}_{n}=\sup _{n} \bar{F}_{n} .
$$

Remark. The proof of this proposition is simple and based just on the exploitation of the definitions (see, for instance, [18: Chapter 5] where it is stated in an even more general setting).

2.2 Regularization and approximating problems. We recall that the question we address in this article is to get an homogenization formula for the family of problems $\left(\mathrm{P}_{\varepsilon}\right)$. We regularize the integrand $f$ in $F_{\varepsilon}$ by infconvolution in order to get an approximating family of integrands $\left(f_{\lambda}\right)_{\lambda>0}$ satisfying standard growth conditions of order $N+1$. Each function $f_{\lambda}$ is given by

$$
f_{\lambda}(x, \xi)=\inf \left\{f(x, \zeta)+\lambda|\xi-\zeta|^{N+1}: \zeta \in \mathbb{R}^{N}\right\}
$$

for all $(x, \xi)$ where $\lambda$ is a positive parameter intended to go to $+\infty$. The main properties of this new integrand are the following:

(1) For any $x$ and $\xi, \max \{0,|\xi|-R\}^{N+1} \leq f_{\lambda}(x, \xi) \leq f(x, 0)+\lambda|\xi|^{N+1}$.

(2) $f_{\lambda}(x, \xi) \rightarrow f(x, \xi)$ increasingly when $\lambda \rightarrow \infty$.

The main consequence of the first property is that the associated functionals $F_{\varepsilon, \lambda}$ given on $\mathcal{C}_{0}(\Omega)$ by

$$
F_{\varepsilon, \lambda}(v)= \begin{cases}\int_{\Omega} f_{\lambda}\left(\frac{x}{\varepsilon}, D v(x)\right) d x & \text { if } v \in W^{1, N+1}\left(\Omega ; \mathbb{R}^{N}\right) \cap \mathcal{C}_{0}(\Omega) \\ +\infty & \text { elsewhere }\end{cases}
$$

is coercive as well as lower semicontinuous on $\mathcal{C}_{0}(\Omega)$.

The approximating energies associated to each $F_{\varepsilon}$ we will then consider are the functionals $F_{\varepsilon, \lambda}$ defined by (2.3) for positive real numbers $\lambda$. The approximating problems are thus

$$
\left(\mathbf{P}_{\varepsilon, \lambda}\right) \inf \left\{F_{\varepsilon, \lambda}(v)+\int_{\Omega} \beta(x) v(x) d x: v \in \mathcal{C}_{0}(\Omega)\right\} .
$$


As we shall see, the monotonicity of this regularization scheme will be of help in the following proofs.

2.3 The classical periodic homogenization formula. In this subsection we report a classical result in the periodic homogenization theory. This part of the theory has been widely developed in the literature (having to select a reference we refer to the recent book [5: Chapter 14]).

Define $G_{\varepsilon}: \mathcal{C}_{0}(\Omega) \rightarrow \mathbb{R}$ as

$$
G_{\varepsilon}(v)= \begin{cases}\int_{\Omega} g\left(\frac{x}{\varepsilon}, D v(x)\right) d x & \text { if } v \in W^{1, p}(\Omega) \cap \mathcal{C}_{0}(\Omega) \\ +\infty & \text { elsewhere }\end{cases}
$$

where $N<p<+\infty$ and the integrand $g$ is a Borel function which satisfies the following conditions:

- (Periodicity) $g(\cdot, \xi)$ is $(0,1)^{N}$-periodic for all $\xi$.

- (Convexity) $g(x, \cdot)$ is lower semicontinuous and convex for all $x$.

- (Standard $p$-growth conditions) There exist $0<a \leq b$ such that $a|\xi|^{p} \leq$ $g(x, \xi) \leq b\left(1+|\xi|^{p}\right)$ for all $x$ and $\xi$.

Under the above assumptions, the following theorem holds.

Theorem 2.3. The functionals $G_{\varepsilon} \Gamma$-converge in $\mathcal{C}_{0}(\Omega)$ as $\varepsilon \rightarrow 0$ to $G_{\text {hom }}$, with

$$
G_{\mathrm{hom}}(v)= \begin{cases}\int_{\Omega} g_{\mathrm{hom}}(D v(x)) d x & \text { if } v \in W^{1, p}(\Omega) \cap \mathcal{C}_{0}(\Omega) \\ +\infty & \text { elsewhere }\end{cases}
$$

where $g_{\mathrm{hom}}: \mathbb{R}^{N} \mapsto \mathbb{R}_{+}$is given for $\xi \in \mathbb{R}^{N}$ by the formula

$$
\begin{aligned}
& g_{\text {hom }}(\xi)= \\
& \inf \left\{\int_{(0,1)^{N}} g(x, \xi+D v(x)) d x: v \in W_{\#}^{1, p}\left((0,1)^{N}\right) \cap \mathcal{C}\left((0,1)^{N}\right)\right\} .
\end{aligned}
$$

Remark. In the above formula, $W_{\sharp}^{1, p}\left((0,1)^{N}\right)$ denotes the set of functions of $W_{l o c}^{1, p}\left(\mathbb{R}^{N}\right)$ which are $(0,1)^{N}$-periodic.

As a consequence, we get the following homogenization results for the approximating functionals $F_{\varepsilon, \lambda}$ for fixed $\lambda>0$.

Corollary 2.1. For any $\lambda>0$, the family of functionals $\left(F_{\varepsilon, \lambda}\right)_{\varepsilon} \Gamma-$ converges to $F_{\text {hom }}$ with

$$
F_{h o m, \lambda}(v)= \begin{cases}\int_{\Omega} f_{h o m, \lambda}(D v(x)) d x & \text { if } v \in W^{1, N+1}(\Omega) \cap \mathcal{C}_{0}(\Omega) \\ +\infty & \text { elsewhere }\end{cases}
$$


for the uniform convergence in $\mathcal{C}_{0}(\Omega)$ and where $f_{\text {hom, } \lambda}$ is given by

$$
\begin{aligned}
& f_{\text {hom }, \lambda}(\xi)= \\
& \inf \left\{\int_{(0,1)^{N}} f_{\lambda}(y, \xi+D v(y)) d y: v \in W_{\#}^{1, N+1}\left((0,1)^{N}\right) \cap \mathcal{C}\left((0,1)^{N}\right)\right\} .
\end{aligned}
$$

\section{Main results}

The main result of this paper is the following $\Gamma$-convergence property for the family $\left(F_{\varepsilon}\right)_{\varepsilon>0}$.

Theorem 3.1. Assume that hypotheses (1) - (3) as well as (1.1) hold. Then the family of functionals $\left(F_{\varepsilon}\right)_{\varepsilon>0} \Gamma$-converges in $\mathcal{C}_{0}(\Omega)$ to the homogenized functional $F_{\text {hom }}$.

As we shall see in the proof of the above theorem (see Section 4), the $\Gamma$-liminf estimate on the family $\left(F_{\varepsilon}\right)_{\varepsilon}$ does not require hypothesis $(1.1)$, which is only necessary for the proof of the upper bound for the $\Gamma$-limsup.

Since the family $\left(F_{\varepsilon}\right)_{\varepsilon}$ is equicoercive on $\mathcal{C}_{0}(\Omega)$, we infer from Theorems 2.1 and 3.1 the following result that is the homogenization property discussed in the introduction.

Theorem 3.2. Assume that hypotheses (1) - (3) and (1.1) hold. Then the family of minima $\left(\min \left(\mathrm{P}_{\varepsilon}\right)\right)_{\varepsilon}$ of problems $\left(\mathrm{P}_{\varepsilon}\right)$ converges as $\varepsilon$ goes to 0 to the minimum $\min \left(\mathrm{P}_{\text {hom }}\right)$ of problem $\left(\mathrm{P}_{\text {hom }}\right)$. Moreover, if for each $\varepsilon>0$ the function $u_{\varepsilon}$ is a minimizer of $\left(\mathrm{P}_{\varepsilon}\right)$ and if $\left(u_{\varepsilon_{k}}\right)_{k}$ converges in $\mathcal{C}_{0}(\Omega)$ to some function $u_{\mathrm{hom}}$, then $u_{\mathrm{hom}}$ is a minimizer of problem $\left(\mathrm{P}_{\mathrm{hom}}\right)$ and

$$
\begin{aligned}
\lim _{k} & \left(F_{\varepsilon}\left(u_{\varepsilon_{k}}\right)+\int_{\Omega} \beta(x) u_{\varepsilon_{k}}(x) d x\right) \\
& =F_{\mathrm{hom}}\left(u_{\mathrm{hom}}\right)+\int_{\Omega} \beta(x) u_{\mathrm{hom}}(x) d x \\
& =\min \left(P_{\text {hom }}\right) .
\end{aligned}
$$

Remark. In the above result, the linear functional $v \mapsto \int_{\Omega} \beta(x) v(x) d x$ may of course be replaced by any continuous functional $v \mapsto F(v)$ on $C_{0}(\Omega)$.

In [16], a $\Gamma$-convergence result analogous to Theorem 3.1 is obtained under more restrictive hypotheses than $(1)-(3)$, while hypothesis (1.1) is replaced by its part a), i.e.

0 belongs to the interior of $\operatorname{dom}\left(f_{\text {hom }}\right)$. 
In [19], Theorem 3.1 is also shown under slightly more restrictive hypotheses than those assumed here, and (1.1) is replaced by the following alternative:

either (3.1) holds

or the interior of $C_{\text {hom }}$ is empty and part b) of (1.1) holds.

We notice that part $b$ ) of (1.1) may be satisfied even if the interior of $C_{\text {hom }}$ is empty. For instance, take $f(x, \xi)=|\xi|^{2}+\delta_{\left[0,1\left[^{N}\right.\right.}(\xi)$ for any $x$ and $\xi$. Then $C_{\text {hom }}=\left[0,1\left[^{N}\right.\right.$ and $\left.b\right)$ of $(1.1)$ holds with $w \equiv 0$.

\section{Proof of Theorem 3.1}

We split the proof of the $\Gamma$-convergence result into two parts: the first one, which is devoted to the proof of the lower bound on the $\Gamma$-liminf, is mainly based on Theorem 4.2 and the properties of the Moreau-Yosida approximation, while in the second part the upper bound for the $\Gamma$-limsup is obtained by more classical approximation arguments.

4.1 Proof of the lower bound $\Gamma$-liminf $\boldsymbol{F}_{\boldsymbol{\varepsilon}} \geq \boldsymbol{F}_{\boldsymbol{h o m}}$. This subsection is devoted to the following lower bound for the $\Gamma$-liminf of the family $\left(F_{\varepsilon}\right)_{\varepsilon}$.

Theorem 4.1. Assume that hypotheses (1)-(3) hold. Then $\Gamma-\liminf _{\varepsilon} F_{\varepsilon}$ $\geq F_{\text {hom }}$.

The corner stone to prove this theorem is the following $\Gamma$-convergence result for the approximating functionals $F_{\varepsilon, \lambda}$. Its proof mainly relies on the fact that the approximation scheme $\lambda \mapsto f_{\lambda}$ is monotone.

Theorem 4.2. Assume that hypotheses (1) - (3) hold. Then for every $\varepsilon>0$ the family of functionals $\left(F_{\varepsilon, \lambda}\right)_{\lambda>0} \Gamma$-converges in $\mathcal{C}_{0}(\Omega)$ to $F_{\varepsilon}$ as $\lambda \rightarrow$ $\infty$. Moreover, one has

$$
\Gamma-\lim _{\lambda}\left(\Gamma-\lim _{\varepsilon} F_{\varepsilon, \lambda}\right)=\Gamma-\lim _{\lambda} F_{h o m, \lambda}=F_{\text {hom }}
$$

where the $\Gamma$-limit is taken for the uniform convergence norm on $\mathcal{C}_{0}(\Omega)$.

Since for any $\lambda>0$ and $\varepsilon>0$ one has $F_{\varepsilon} \geq F_{\varepsilon, \lambda}$ on $\mathcal{C}_{0}(\Omega)$, we easily infer from Theorem 4.2 that

$$
\Gamma-\liminf _{\varepsilon} F_{\varepsilon} \geq \Gamma-\liminf _{\lambda}\left(\Gamma-\liminf _{\varepsilon} F_{\varepsilon, \lambda}\right)=F_{\text {hom }}
$$

where the $\Gamma$-limits are taken for the uniform convergence norm on $\Omega$. It thus remains to prove Theorem 4.2.

We begin with a result on the $\Gamma$-convergence of non-decreasing families of integral functionals. This result is not optimal but it is sufficient for our purpose, and its proof is given here for the sake of completeness. 
Proposition 4.3. Let $\omega$ be a bounded open subset of $\mathbb{R}^{N}$, and $\left(g_{\lambda}\right)_{\lambda>0}$ be a non-decreasing family of functions from $\omega \times \mathbb{R}^{N}$ to $[0,+\infty)$ for which there is a $p>N$ with the property: for all $\lambda>0$ there exists $a b_{\lambda} \geq a_{\lambda}>0$ such that

$$
a_{\lambda}\left(|\xi|^{p}-1\right) \leq g_{\lambda}(x, \xi) \leq b_{\lambda}\left(|\xi|^{p}+1\right)
$$

for all $x$ and $\xi$. We assume that the integral functionals $G_{\lambda}$ defined on $\mathcal{C}(\bar{\omega})$ by

$$
G_{\lambda}(v)= \begin{cases}\int_{\Omega} g_{\lambda}(x, D v(x)) d x & \text { if } v \in X \\ +\infty & \text { otherwise }\end{cases}
$$

are lower semicontinuous on $X$, where $X$ is a closed subspace $\left(\right.$ in $W^{1, p}(\omega)$ ) of $W^{1, p}(\omega) \cap \mathcal{C}(\bar{\omega})$. Let $g_{\infty}: \omega \times \mathbb{R}^{N} \rightarrow \mathbb{R} \cup\{+\infty\}$ denote the pointwise limit (as $\lambda$ tends to infinity) of $\left(g_{\lambda}\right)_{\lambda>0}$ and $G_{\infty}$ the associated functional on $\mathcal{C}(\bar{\omega})$ given by

$$
G_{\infty}(v)= \begin{cases}\int_{\Omega} g_{\infty}(x, D v(x)) d x & \text { if it is finite and } v \in X \\ +\infty & \text { otherwise. }\end{cases}
$$

Then the family of functionals $\left(G_{\lambda}\right)_{\lambda>0} \Gamma$-converges to $G_{\infty}$ in $\mathcal{C}(\bar{\omega})$.

Proof. Since the family $\left(G_{\lambda}\right)_{\lambda>0}$ is non-decreasing on $\mathcal{C}(\bar{\omega})$, we conclude from Proposition 2.2 that

$$
\Gamma-\lim _{\lambda \rightarrow+\infty} G_{\lambda}=\sup _{\lambda>0} G_{\lambda}
$$

where the $\Gamma$-limit is taken for the uniform convergence on $\Omega$. It thus remains to prove that $G_{\infty}=\sup _{\lambda} G_{\lambda}$. For this, let $v$ belong to $\mathcal{C}(\bar{\omega})$. Then if $G_{1}(v)=$ $+\infty$, one gets $\sup _{\lambda} G_{\lambda}(v)=+\infty=G_{\infty}(v)$, which is our claim. Otherwise, the function $g_{1}(\cdot, D v(\cdot))$ belongs to $L^{1}(\Omega)$, so that we can apply Lebesgue's monotone convergence theorem to the non-negative and non-decreasing family $\left(g_{\lambda}(\cdot, D v(\cdot))-g_{N+1}(\cdot, D v(\cdot))\right)_{\lambda>0}$, which yields

$$
\lim _{\lambda \rightarrow+\infty} G_{\lambda}(v)=\sup _{\lambda>0} G_{\lambda}(v)=G_{\infty}(v) .
$$

This concludes the proof

As consequence of Proposition 4.3 we easily get the first part of Theorem 4.2 , that is

$$
\Gamma-\lim _{\lambda \rightarrow+\infty} F_{\varepsilon, \lambda}=F_{\varepsilon}
$$

where the $\Gamma$-limit is taken for the uniform convergence norm on $\mathcal{C}_{0}(\Omega)$. Proposition 4.3 also yields the following convergence result. 
Lemma 4.4. The family $\left(f_{h o m, \lambda}\right)_{\lambda>0}$ is non-decreasing on $\mathbb{R}^{N}$ and converges to $f_{\text {hom }}$ as $\lambda$ tends to $+\infty$.

Proof. The monotonicity of the family $\left(f_{h o m, \lambda}\right)_{\lambda>0}$ follows from that of $f_{\lambda}$. For any $\xi$ in $\mathbb{R}^{N}, \lambda>0$ and $(y, \zeta)$ in $(0,1)^{N} \times \mathbb{R}^{N}$, we set

$$
\begin{aligned}
g_{\lambda}^{\xi}(y, \zeta) & =f_{\lambda}(y, \xi+\zeta) \\
g_{\infty}^{\xi}(y, \zeta) & =f(y, \xi+\zeta)
\end{aligned}
$$

and define the associated functionals on $\mathcal{C}\left((0,1)^{N}\right)$

$$
\begin{aligned}
& G_{\lambda}^{\xi}(v)= \begin{cases}\int_{(0,1)^{N}} g_{\lambda}^{\xi}(y, D v(y)) d y & \text { when }\left\{\begin{array}{c}
v \in W_{\#}^{1, N+1}\left((0,1)^{N}\right) \\
\cap \mathcal{C}\left((0,1)^{N}\right) \\
\int_{(0,1)^{N}} v(x) d x=0
\end{array}\right. \\
\text { otherwise }\end{cases} \\
& G_{\infty}^{\xi}(v)= \begin{cases}\int_{(0,1)^{N}} g_{\infty}^{\xi}(y, D v(y)) d y & \text { when }\left\{\begin{array}{c}
v \in W_{\#}^{1, \infty}\left((0,1)^{N}\right) \\
\cap \mathcal{C}\left((0,1)^{N}\right) \\
\int_{(0,1)^{N}} v(x) d x=0
\end{array}\right. \\
\text { otherwise. }\end{cases}
\end{aligned}
$$

With these notations, $f_{\text {hom }, \lambda}(\xi)=\inf \left(G_{\lambda}^{\xi}\right)$ as well as $f_{\text {hom }}(\xi)=\inf \left(G_{\infty}^{\xi}\right)$. Notice that each functional $G_{\lambda}^{\xi}$ is lower semicontinuous on $\mathcal{C}\left((0,1)^{N}\right)$. Since the family $\left(g_{\lambda}^{\xi}\right)_{\lambda>0}$ is non-decreasing and converges to $g_{\infty}^{\xi}$, we can apply Proposition 4.3 so that the family $\left(G_{\lambda}^{\xi}\right)_{\lambda>0} \Gamma$-converges to $G_{\text {hom }}^{\xi}$ in $\mathcal{C}\left((0,1)^{N}\right)$. Since the family $\left(G_{\lambda}^{\xi}\right)_{\lambda>0}$ is equicoercive, we deduce from Theorem 2.1 that the family $\left(f_{\text {hom }, \lambda}(\xi)\right)_{\lambda>0}$ converges to $f_{\text {hom }}(\xi)$, and the proof is complete

As consequence of Proposition 4.3 and Lemma 4.4, the family $\left(F_{h o m, \lambda}\right)_{\lambda>0}$ $\Gamma$-converges in $\mathcal{C}_{0}(\Omega)$ to $F_{\text {hom }}$ as $\lambda$ tends to $+\infty$. This concludes the proof of Theorem 4.2 .

4.2 Proof of the upper bound $\boldsymbol{\Gamma}$-limsup $\boldsymbol{F}_{\varepsilon} \leq \boldsymbol{F}_{\boldsymbol{h} o m}$. We now prove the upper bound on the $\Gamma$-limsup of the family $\left(F_{\varepsilon}\right)_{\varepsilon}$, which is stated as follows.

Theorem 4.5. Assume that hypotheses (1) - (3) as well as hypothesis (1.1) hold. Then $\Gamma-\lim \sup _{\varepsilon} F_{\varepsilon} \leq F_{\text {hom }}$.

To prove this theorem, we introduce the functional $G$, given on $\mathcal{C}_{0}(\Omega)$ by

$$
G(v)= \begin{cases}\int_{\Omega} g(D v(x)) d x & \text { if finite and } v \text { in } W^{1, \infty}(\Omega) \cap \mathcal{C}_{0}(\Omega) \\ +\infty & \text { elsewhere }\end{cases}
$$


where the integrand $g$ is defined on $\mathbb{R}^{N}$ by $g(\xi)=f_{\text {hom }}(\xi)$ if $\xi \neq 0$ and

$$
g(0)=\inf \left\{\int_{(0,1)^{N}} f(y, D v(y)) d y: v \in W^{1, \infty}\left((0,1)^{N}\right) \cap \mathcal{C}_{0}\left((0,1)^{N}\right)\right\} .
$$

We notice that the integrand $g$ obviously has the same domain as $f_{\text {hom }}$, and we shall denote this domain $C_{\text {hom }}$ in the following. Notice that $C_{\text {hom }}$ is a bounded convex subset of $\mathbb{R}^{N}$. We also point out that, for any $\xi$ in $C_{\text {hom }}$, the infimum in the definition of $f_{\text {hom }}$ ( or $g$ ) is attained thanks to hypothesis $(3)$.

Remark. If part $a)$ of (1.1) holds, then $F_{\text {hom }} \leq G$ while if part $b$ ) of (1.1) holds, then $F_{\text {hom }}=G$.

We now proceed in three steps for the proof of Theorem 4.5. First we prove that $\Gamma-\lim \sup F_{\varepsilon}(u) \leq G(u)$ for the function $u=u^{0}$ where $u^{0}$ denotes the null function on $\Omega$. Then we notice that if 0 is not in the interior of $C_{\text {hom }}$, then the theorem follows from hypothesis (1.1). Otherwise, when $0 \in \operatorname{int}\left(C_{\mathrm{hom}}\right)$, we extend the inequality $\Gamma$-limsup $F_{\varepsilon}(u) \leq G(u)$ to the functions $u$ which are continuous, piecewise affine and have compact support in $\Omega$. What we mean by " $u$ is continuous, piecewise affine and has compact support in $\Omega$ " is that $u$ belongs to $\mathcal{C}_{0}(\Omega)$ and there exists a finite family $\left(K_{i}\right)_{1 \leq i \leq n}$ of disjoint open sets in $\Omega$ such that $\cup_{\{1 \leq i \leq n\}} K_{i}$ is relatively compact in $\Omega, u$ is affine on each $K_{i}$ and $u$ is null on $K_{0}=\Omega \backslash \cup_{\{1 \leq i \leq n\}} \overline{K_{i}}$. We then infer $\Gamma$-lim sup $F_{\varepsilon}(u) \leq F_{\text {hom }}(u)$ for any such function $u$, and finally we prove it for any function $u$ in the domain of $F_{\text {hom }}$ (there is nothing to prove for functions $u$ for which $F_{\text {hom }}(u)=+\infty$ ). The proofs of these steps consist in the following serie of lemmas, which hold under the same assumptions as those of Theorem 4.5.

Lemma 4.6. We have $\left(\Gamma-\lim \sup F_{\varepsilon}\right)\left(u^{0}\right) \leq G\left(u^{0}\right)$, where $u^{0}$ denotes the null function on $\Omega$.

Proof. Let $w^{0}$ in $W^{1, \infty}\left((0,1)^{N}\right) \cap \mathcal{C}_{0}\left((0,1)^{N}\right)$ be such that

$$
g(0)=\int_{(0,1)^{N}} f\left(y, D w^{0}(y)\right) d y
$$

For each number $\varepsilon>$, we set

$$
\begin{aligned}
& Z_{\varepsilon}=\left\{z \in \mathbb{Z}^{N}: \varepsilon\left(z+(0,1)^{N}\right) \subset \Omega\right\} \\
& \Omega_{\varepsilon}=\Omega \backslash\left(\cup_{\left\{z \in Z_{\varepsilon}\right\}} \varepsilon\left(z+[0,1]^{N}\right)\right) .
\end{aligned}
$$

We notice that since $\Omega$ is open, $\left|\Omega_{\varepsilon}\right| \rightarrow 0$ as $\varepsilon \rightarrow 0$. We also set

$$
R^{\varepsilon}=\left\{z \in \mathbb{Z}^{N}: \varepsilon\left(z+(0,1)^{N}\right) \cap \Omega_{\varepsilon} \neq \emptyset\right\}
$$


and we notice that $\cup_{\left\{z \in R^{\varepsilon}\right\}} \varepsilon\left(z+[0,1]^{N}\right)$ is included in $\Omega_{\varepsilon}+B\left(0, \varepsilon^{1 / N}\right)$. We now consider the family $\left(u_{\varepsilon}^{0}\right)_{\varepsilon}$ of functions of $W^{1, \infty}(\Omega) \cap \mathcal{C}_{0}(\Omega)$ given by

$$
u_{\varepsilon}^{0}(x)= \begin{cases}\varepsilon w^{0}\left(\frac{x-\varepsilon z}{\varepsilon}\right) & \text { if, for some } z \in Z_{\varepsilon}, x \in \varepsilon\left(z+(0,1)^{N}\right) \\ 0 & \text { if } x \in \Omega_{\varepsilon} .\end{cases}
$$

This family converges in $\mathcal{C}_{0}(\Omega)$ to $u^{0}$, and we have

$$
\begin{aligned}
F_{\varepsilon}\left(u_{\varepsilon}^{0}\right) & =\int_{\Omega_{\varepsilon}} f\left(\frac{x}{\varepsilon}, 0\right) d x+\sum_{z \in Z_{\varepsilon}} \varepsilon^{N} \int_{(0,1)^{N}} f\left(y, D w^{0}(y)\right) d y \\
& \leq \sum_{z \in R^{\varepsilon}} \varepsilon^{N} \int_{(0,1)^{N}} f(y, 0) d y+\left|\cup_{\left\{z \in Z_{\varepsilon}\right\}} \varepsilon\left(z+(0,1)^{N}\right)\right| g(0) \\
& \leq\left|\Omega_{\varepsilon}+B(0, \sqrt{N} \varepsilon)\right| \int_{(0,1)^{N}} f(y, 0) d y+|\Omega| g(0)
\end{aligned}
$$

Since $\left|\Omega_{\varepsilon}+B(0, \sqrt{N} \varepsilon)\right|$ goes to 0 as $\varepsilon$ goes to 0 and $f(\cdot, 0)$ is in $L^{1}\left((0,1)^{N}\right)$, we infer that $\lim \sup F_{\varepsilon}\left(u_{\varepsilon}^{0}\right) \leq|\Omega| g(0)=G\left(u^{0}\right)$, which concludes the proof

Lemma 4.7. Assume that $0 \notin \operatorname{int}\left(C_{\mathrm{hom}}\right)$. Then the domain of the functional $F_{\text {hom }}$ reduces to $u^{0}$.

Proof. Since 0 belongs to the boundary of the convex set $C_{\mathrm{hom}}$, there exists $\xi$ in $\mathbb{R}^{N} \backslash\{0\}$ such that $\langle\xi, \zeta\rangle \leq 0$ for every $\zeta$ in $C_{\text {hom }}$. Letting $u \in$ $W^{1, \infty}(\Omega) \cap \mathcal{C}_{0}(\Omega)$ be such that $F_{\text {hom }}(u)<+\infty$, we want to show that $u=u^{0}$. Since $u=0$ on $\partial \Omega$, we have $\int_{\Omega} D u(x) d x=0$ and thus $\int_{\Omega}\langle D u(x), \xi\rangle d x=0$. But as $F_{\text {hom }}(u)<+\infty, D u(x)$ belongs to $C_{\text {hom }}$ for a.e. $x$ in $\Omega$, so that $\langle D u(x), \xi\rangle=0$ for a.e. $x \in \Omega$.

Supposing that $u \not \equiv 0$, we extend $u$ on $\mathbb{R}^{N}$ by $u=0$ on $\mathbb{R}^{N} \backslash \Omega$, and consider $u^{\delta}=\gamma_{\delta} * u$, where $\delta$ is a positive real number and $\gamma_{\delta}$ is a mollifier, i.e. $\gamma_{\delta}(x)=\delta^{N} \gamma\left(\frac{x}{\delta}\right)$ for some function $\gamma$ such that

$$
\gamma \in C^{\infty}\left(\mathbb{R}^{N},[0,+\infty)\right), \quad \operatorname{supp}(\gamma) \subset B(0,1), \quad \int_{\mathbb{R}^{N}} \gamma(x) d x=1 .
$$

Since $u \not \equiv 0$, for $\delta$ small enough there exists $y$ in $\mathbb{R}^{N}$ such that $u^{\delta}(y) \neq 0$. If we define $v$ on $\mathbb{R}$ by $v(t)=u^{\delta}(y+t \xi)$ for such $\delta$ and $y$, then $v$ has compact support and

$$
v^{\prime}(t)=\left\langle D u^{\delta}(y+t \xi), \xi\right\rangle=\int_{\mathbb{R}^{N}} \gamma_{\delta}(y+t \xi-x)\langle D u(x), \xi\rangle d x=0
$$

for all $t \in \mathbb{R}$ so that $v$ is constant on $\mathbb{R}$ and hence null, which contradicts $v(0)=u^{\delta}(y) \neq 0$ 
If 0 does not belong to the interior of $C_{\text {hom }}$, then we deduce from hypothesis (1.1) that $g(0)=f_{\text {hom }}(0)$ so that $G\left(u^{0}\right)=F_{\text {hom }}\left(u^{0}\right)$. Then Lemmas 4.6 and 4.7 yield that Theorem 4.5 holds in this case.

Remark. From now on we thus assume that 0 belongs to the interior of $C_{\text {hom }}$.

Lemma 4.8. Assume that $u$ is continuous, piecewise affine and has compact support in $\Omega$. Then $\left(\Gamma-\lim \sup F_{\varepsilon}\right)(u) \leq G(u)$.

Proof. Let $\left(K^{i}\right)_{1 \leq i \leq n}$ be a finite family of disjoint open sets such that $\cup_{\{1 \leq i \leq n\}} K^{i}$ is relatively compact in $\Omega, u$ is affine on each $K^{i}$ and $u$ is null on $K^{0}=\Omega \backslash \cup_{1 \leq i \leq n} \overline{K^{i}}$. Then, for each $i \in\{1, \ldots, n\}$, there exist $\alpha_{i}$ in $\mathbb{R}$ and $\xi_{i}$ in $\mathbb{R}^{N}$ such that $u(x)=\xi_{i} \cdot x+\alpha_{i}$ for any $x$ in $K^{i}$ (of course, we set $\xi_{0}=0$ and $\left.\alpha_{0}=0\right)$. As a consequence, one has $G(u)=\sum_{\{0 \leq i \leq n\}}\left|K^{i}\right| g\left(\xi_{i}\right)$. In the sequel, we assume that $G(u)<+\infty$, otherwise there is nothing to prove. Moreover, we assume that $\left(\xi_{i}, \alpha_{i}\right) \neq\left(\xi_{j}, \alpha_{j}\right)$ for $i \neq j$.

For indices $i \in\{0, \ldots, n\}$ such that $\xi_{i}=0$, we set $u_{\varepsilon}^{i}=\alpha_{i}+u_{\varepsilon}^{0}$ for any $\varepsilon>0$, where the family of functions $\left(u_{\varepsilon}^{0}\right)_{\varepsilon}$ is that given in Lemma 4.6. For the indices $i$ in $\{1, \ldots, n\}$ for which $\xi_{i} \neq 0$, we take $w^{i}$ in $W_{\#}^{1, \infty}\left((0,1)^{N}\right) \cap$ $\mathcal{C}\left((0,1)^{N}\right)$ so that

$$
g\left(\xi_{i}\right)=\int_{(0,1)^{N}} f\left(y, \xi_{i}+D w^{i}(y)\right) d y
$$

For such indices $i$ and $\varepsilon>0$, we define the function $u_{\varepsilon}^{i} \in W^{1, \infty}(\Omega) \cap \mathcal{C}(\Omega)$ by

$$
u_{\varepsilon}^{i}(x)=\xi_{i} \cdot x+\alpha_{i}+\varepsilon w^{i}\left(\frac{x-\varepsilon z}{\varepsilon}\right) \quad \text { if }\left\{\begin{array}{l}
x \in \varepsilon\left(z+(0,1)^{N}\right) \\
\text { for some } z \in \mathbb{Z}^{N} .
\end{array}\right.
$$

Then, for any $i$ in $\{0, \ldots, n\}$, the family $\left(u_{\varepsilon}^{i}\right)_{\varepsilon}$ converges in $\mathcal{C}(\Omega)$ to $u^{i}$, where $u^{i}$ is defined on $\Omega$ by $u^{i}(x)=\xi_{i} \cdot x+\alpha_{i}$.

Since the function $u$ has compact support in $\Omega$, we can extend it on $\mathbb{R}^{N}$ by $u \equiv 0$ on $\mathbb{R}^{N} \backslash \Omega$. We may thus apply [10: Theorem 2.1] which state that the real-valued continuous piecewise affine function $u$ may be written as a finite combination of "min" and "max" of its affine components. We shall formally denote $u=c\left(u^{0}, \ldots, u^{n}\right)$ where $c$ is a combination of "min" and "max" operators. For any positive $\varepsilon$, we then define the function $u_{\varepsilon} \in \mathcal{C}(\Omega)$ through the same combination $u_{\varepsilon}=c\left(u_{\varepsilon}^{0}, \ldots, u_{\varepsilon}^{n}\right)$. We now set

$$
K_{\varepsilon}^{i}=\left\{x \in \Omega: u_{\varepsilon}(x)=u_{\varepsilon}^{i}(x)\right\} \backslash \cup_{\{j<i\}} K_{\varepsilon}^{j}
$$

for any $\varepsilon>0$ and $i \in\{0, \ldots, n\}$. We notice that $D u_{\varepsilon}(x)=D u_{\varepsilon}^{i}(x)$ for a.e. $x$ in $K_{\varepsilon}^{i}$. Moreover, $u_{\varepsilon}=0$ on $\partial \Omega$ for $\varepsilon$ small enough, so that

$$
F_{\varepsilon}\left(u_{\varepsilon}\right)=\sum_{i=0}^{n} \int_{K_{\varepsilon}^{i}} f\left(\frac{x}{\varepsilon}, D u_{\varepsilon}^{i}(x)\right) d x .
$$


The same argument as in the proof of Lemma 4.6 then yields

$$
\begin{aligned}
\left|\cup_{\left\{z \in Z_{\varepsilon}^{i}\right\}} \varepsilon\left(z+(0,1)^{N}\right)\right| g\left(\xi_{i}\right) & \leq \int_{K_{\varepsilon}^{i}} f\left(\frac{x}{\varepsilon}, D u_{\varepsilon}^{i}(x)\right) d x \\
& \leq\left|K_{\varepsilon}^{i}+B\left(0, \varepsilon^{1 / N}\right)\right| g\left(\xi_{i}\right)
\end{aligned}
$$

for any $\varepsilon>0$ and $i \in\{1, \ldots, n\}$, where

$$
Z_{\varepsilon}^{i}=\left\{z \in \mathbb{Z}^{N}: \varepsilon\left(z+(0,1)^{N}\right) \subset K_{\varepsilon}^{i}\right\} .
$$

Since the families of functions $\left(u_{\varepsilon}^{i}\right)_{\varepsilon}$ converge uniformly on $\bar{\Omega}$ to $u^{i}$ for any $i$, we infer that $\left(u_{\varepsilon}\right)_{\varepsilon}$ converges uniformly on $\bar{\Omega}$ to $u$ and that the families of $\left(\left|K_{\varepsilon}^{i}\right|\right)_{\varepsilon}$ converge to $\left|K^{i}\right|$ for any $i$. We then infer from (4.3) that, for any index $i \in\{1, \ldots, n\}$,

$$
\limsup _{\varepsilon \rightarrow 0} \int_{K_{\varepsilon}^{i}} f\left(\frac{x}{\varepsilon}, D u_{\varepsilon}^{i}(x)\right) d x \leq\left|K^{i}\right| g\left(\xi_{i}\right) .
$$

The same inequality also holds for the index $i=0$, by the same argument as in the proof of Lemma 4.6. Therefore, identity (4.2) yields $\lim \sup F_{\varepsilon}\left(u_{\varepsilon}\right) \leq$ $G(u)$, which concludes the proof

Lemma 4.9. Let 0 belong to the interior of $C_{\mathrm{hom}}$. If $u$ is continuous, piecewise affine and has compact support in $\Omega$, then $\left(\Gamma-\lim \sup F_{\varepsilon}\right)(u) \leq$ $F_{\text {hom }}(u)$.

Proof. Let $u$ be continuous, piecewise affine, with compact support in $\Omega$ and such that $F_{\text {hom }}(u)<+\infty$. As in the proof of Lemma $4.8,\left(K^{i}\right)_{1 \leq i \leq n}$ is a finite family of disjoint open sets such that $\cup_{\{1 \leq i \leq n\}} K^{i}$ is relatively compact in $\Omega, u$ is affine on each $K^{i}$ and $u$ is null on $K^{0}=\Omega \backslash \cup_{1 \leq i \leq n} \overline{K^{i}}$. We shall then write $u(x)=\xi_{i} \cdot x+\alpha_{i}$ for any $x \in K^{i}$. We claim that there exists a sequence of continuous, piecewise affine functions $\left(u_{n}\right)_{n}$ with compact support in $\Omega$ which converges to $u$ in $\mathcal{C}_{0}(\Omega)$ and for which $\lim _{n \rightarrow+\infty} G\left(u_{n}\right)=F_{\text {hom }}(u)$.

Setting $I=\left\{i: \xi_{i}=0\right\}$ and $J=\{0, \ldots, n\} \backslash I$, we notice that for $j \in J$ one has $g\left(\xi_{j}\right)=f_{\text {hom }}\left(\xi_{j}\right)$. Further, for any $i \in I$ and $n \in \mathbb{N}^{*}$ we set

$$
\begin{aligned}
Z_{n}^{i} & =\left\{z \in \mathbb{Z}^{N}: \frac{1}{n}\left(z+[0,1]^{N}\right) \subset K^{i}\right\} \\
\Omega_{n} & =\bigcup_{\{i \in I\}} \bigcup_{\left\{z \in Z_{n}^{i}\right\}} \frac{1}{n}\left(z+[0,1]^{N}\right) .
\end{aligned}
$$

Notice that $\Omega_{n} \subset \subset \Omega$. Let now $w$ be the piecewise affine function on $[0,1]^{N}$ given by

$$
w(x)=w\left(x_{1}, \ldots, x_{N}\right)=\min \left\{x_{1}, \ldots, x_{N}, 1-x_{1}, \ldots, 1-x_{N}\right\},
$$


then $w$ is constant equal to 0 on the boundary of $[0,1]^{N}$ and $0<|D w| \leq 1$ a.e. in $[0,1]^{N}$. For any $n \in \mathbb{N}^{*}$ we define the function $u_{n} \in \mathcal{C}_{0}(\Omega)$ by

$$
u_{n}(x)= \begin{cases}u(x) & \text { if } x \in \Omega \backslash \Omega_{n} \\ u(x)+\frac{1}{n^{2}} w(n x-z) & \text { if } x \in \frac{1}{n}\left(z+[0,1]^{N}\right), z \in Z_{n}^{i} .\end{cases}
$$

For any $n$, the function $u_{n}$ is then continuous, piecewise affine and has compact support in $\Omega$; moreover, the sequence $\left(u_{n}\right)_{n}$ converges to $u$ in $\mathcal{C}_{0}(\Omega)$. Since $D u_{n} \neq 0$ a.e. in $\Omega_{n}$, one has

$$
\begin{aligned}
\int_{K^{i}} g\left(D u_{n}(x)\right) d x & =\left|K^{i} \backslash \Omega_{n}\right| g(0)+\sum_{z \in Z_{n}^{i}} \int_{\frac{1}{n}\left(z+[0,1]^{N}\right)} f_{\mathrm{hom}}\left(D u_{n}(x)\right) d x \\
& =\left|K^{i} \backslash \Omega_{n}\right| g(0)+\left|K^{i} \cap \Omega_{n}\right| \int_{(0,1)^{N}} f_{\mathrm{hom}}\left(\frac{1}{n} D w(x)\right) d x
\end{aligned}
$$

for any $i \in I$. Since $f_{\text {hom }}$ is Lipschitz continuous in a neighborhood of 0 , we deduce from the previous inequality that $\lim _{n \rightarrow+\infty} G\left(u_{n}\right)=F_{\text {hom }}(u)$.

We can now conclude from the lower-semicontinuity of the functional $\Gamma-\lim \sup F_{\varepsilon}$ and from Lemma 4.8 that

$$
\Gamma-\lim \sup F_{\varepsilon}(u) \leq \liminf _{n \rightarrow \infty} \Gamma-\lim \sup F_{\varepsilon}\left(u_{n}\right) \leq \liminf _{n \rightarrow \infty} G\left(u_{n}\right)=F_{\text {hom }}(u)
$$

and the proof is completed

To complete the proof of Theorem 4.5, it thus remains to prove the following

Lemma 4.10. Let 0 belong to $\operatorname{int}\left(C_{\text {hom }}\right)$ and take $u$ in $W^{1, \infty}(\Omega) \cap \mathcal{C}_{0}(\Omega)$ such that $F_{\text {hom }}(u)<+\infty$. Then $\Gamma-\lim \sup F_{\varepsilon}(u) \leq F_{\text {hom }}(u)$.

Proof. From Lemma 4.9, it is sufficient to show that there exists a family $\left(u_{\eta}\right)_{\eta}$ of functions which are continuous, piecewise affine and which have compact support in $\Omega$ and such that

$\left(u_{\eta}\right)_{\eta}$ converges in $\mathcal{C}_{0}(\Omega)$ to $u$

$\lim \sup _{\eta \rightarrow 0} F_{\text {hom }}\left(u_{\eta}\right) \leq F_{\text {hom }}(u)$.

To define such a sequence $\left(u_{\eta}\right)_{\eta}$, we fix some positive real number $\eta$, and proceed in three steps.

First step. For $\alpha$ in $\left(\frac{1}{2}, 1\right)$, we consider the Lipschitz function $\theta_{\alpha}$ given on $\mathbb{R}$ by

$$
\theta_{\alpha}(y)= \begin{cases}0 & \text { if }|y| \leq \frac{1-\alpha}{2} \\ \frac{2}{1+\alpha} y-\operatorname{sign}(y) \frac{1-\alpha}{1+\alpha} & \text { if } \frac{1-\alpha}{2} \leq|y| \leq 1 \\ y & \text { if }|y| \geq 1\end{cases}
$$


Then if we set $v_{\alpha, n}=\frac{1}{n} \theta_{\alpha}(n \alpha u)$, the family $\left(v_{\alpha, n}\right)_{n \in \mathbb{N}}$ converges in $\mathcal{C}_{0}(\Omega)$ to $\alpha u$. Notice that, for every $\alpha$ and $n, v_{\alpha, n}$ has compact support in $\Omega$ since $u \in \mathcal{C}_{0}(\Omega)$. Moreover, for a.e. $x$ in $\Omega, D v_{\alpha, n}(x)=\alpha \theta_{\alpha}^{\prime}(n \alpha u(x)) D u(x)$. But for every $y$ in $\mathbb{R}, \theta_{\alpha}^{\prime}(y)$ belongs to $\left[0, \frac{2}{1+\alpha}\right)$. As a consequence, $D v_{\alpha, n}(y)$ belongs to $\frac{2 \alpha}{1+\alpha} C_{\text {hom }} \subset \subset C_{\text {hom }}$ for a.e. $x$ in $\Omega$. Since $f_{\text {hom }}$ is convex, we get

$$
F_{\text {hom }}\left(v_{\alpha, n}\right) \leq \int_{\Omega} f_{\text {hom }}(D u(x)) d x+(1-\alpha) \int_{\Omega} f_{\text {hom }}(0) d x
$$

so that for $\alpha$ close enough to 1 and $n$ large enough, we have

$$
\begin{aligned}
F_{\text {hom }}\left(v_{\alpha, n}\right) & \leq F_{\text {hom }}(u)+\frac{1}{3} \eta \\
\left\|u-v_{\alpha, n}\right\|_{L^{\infty}(\Omega)} & \leq \frac{1}{3} \eta .
\end{aligned}
$$

We now fix such an $\alpha$ and $n$, and extend $v=v_{\alpha, n}$ by 0 on $\mathbb{R}^{N} \backslash \Omega$.

Second step. For any $\delta>0$ we set $v_{\delta}=\gamma_{\delta} * v$, where $\gamma_{\delta}$ is a mollifier defined as in the proof Lemma 4.7. Since $\operatorname{supp}\left(v_{\delta}\right) \subset \operatorname{supp}(v)+B(0, \delta), v_{\delta}$ has compact support in $\Omega$ for $\delta$ small enough. We infer from the definition of $v$, the convexity of $C_{\text {hom }}$ and from the identity

$$
D v_{\delta}(x)=\int_{\mathbb{R}^{N}} \gamma_{\delta}(x-y) D v(y) d y \quad\left(x \in \mathbb{R}^{N}\right)
$$

that $D v_{\delta}(x)$ also belongs to $\frac{2 \alpha}{1+\alpha} C_{\text {hom }}$ for a.e. $x$ in $\Omega$. Since $f_{\text {hom }}$ is Lipschitz continuous on $\frac{2 \alpha}{1+\alpha} C_{\text {hom }}$, we may choose $\delta$ small enough to have $\| v_{\delta}-$ $v \|_{L^{\infty}(\Omega)} \leq \frac{1}{3} \eta$ as well as $F_{\text {hom }}\left(v_{\delta}\right) \leq F_{\text {hom }}(v)+\frac{1}{3} \eta$.

Third step. For such a positive real number $\delta$, the function $v_{\delta}$ is of class $C^{\infty}$ and has compact support in $\Omega$. It can thus be approximated by a sequence $\left(v_{\delta, k}\right)_{k}$ of continuous and piecewise affine functions with compact support in $\Omega$ and which converge to $v_{\delta}$ in $W^{1, \infty}(\Omega)$. Then for $k$ large enough, $D v_{\delta, k}$ belongs to $\beta C_{\mathrm{hom}}$ a.e. in $\Omega$, for some $\beta \in(0,1)$. Once again we use the fact that $f_{\text {hom }}$ is Lipschitz continuous on $\beta C_{\text {hom }}$, so that for $k$ large enough, the continuous and piecewise affine function $u_{\eta}=v_{\delta, k}$ has compact support in $\Omega$ and satisfies $\left\|v_{\delta}-u_{\eta}\right\|_{L^{\infty}(\Omega)} \leq \frac{1}{3} \eta$ as well as $F_{\text {hom }}\left(u_{\eta}\right) \leq F_{\text {hom }}\left(v_{\delta}\right)+\frac{1}{3} \eta$.

Finally, we thus get a family $\left(u_{\eta}\right)_{\eta>0}$ such that $\left\|u-u_{\eta}\right\|_{L^{\infty}(\Omega)} \leq \eta$ and $F_{\text {hom }}\left(u_{\eta}\right) \leq F_{\text {hom }}(u)+\eta$ for all $\eta>0$. This concludes the proof

Lemmas 4.6 - 4.10 conclude the proof of Theorem 4.5. 


\section{References}

[1] Attouch, H.: Variational Convergence for Functions and Operators (Pitman Advanced Publishing Program). Boston (USA): Pitman 1984.

[2] Attouch, H.: Introduction à l'homogénisation d'inéquations variationnelles. Rend. Sem. Mat. Univ. Politec. Torino 40 (1983), 1 - 23.

[3] Attouch, H. and F. Murat: Homogenization of Fissurated Materials (Publications AVAMAC). Perpignan (France): Université de Perpignan 1985.

[4] Bensoussan, A., Lions, J. L. and G. Papanicolaou: Asymptotic Analysis for Periodic Structures (Studies in Mathematics and its Applications: Vol. 5). Amsterdam: North-Holland 1978.

[5] Braides, A. and A. Defranceschi: Homogenization of Multiple Integrals (Oxford Lecture Series in Mathematics and its Applications: Vol. 12). New York: The Clarendon Press, Oxford University Press 1998.

[6] Carbone, L.: $\Gamma^{-}$-convergence d'intégrales sur des fonctions avec des contraintes sur le gradient. Comm. Part. Diff. Equ. 2 (1977), 627 - 651.

[7] Carbone, L.: The homogenization of a variational problem with constraints on the gradient. Atti Accad. Naz. Lincei Rend. Cl. Sci. Fis. Mat. Natur. (8) 63 (1978), $10-14$.

[8] Carbone, L.: Sur la convergence des intégrales du type de l'énergie sur des fonctions à gradient borné. J. Math. Pures Appl. (9) 56 (1977), 79 - 84.

[9] Carbone, L.: Sur un problème d'homogénéisation avec des contraintes sur le gradient. J. Math. Pures Appl. (9) 58 (1979), 275 - 297.

[10] Carbone, L., Corbo Esposito, A. and R. De Arcangelis: Homogenization of Neumann problems for unbounded integral functionals. Boll. Unione Mat. Ital., Sez. B(8), 2 (1999), $463-491$.

[11] Carbone, L. and S. Salerno: On a problem of homogenization with quickly oscillating constraints on the gradient. J. Math. Anal. Appl. 90 (1982), $219-250$.

[12] Carbone, L. and S. Salerno: Further results on a problem of homogenization with constraints on the gradient. J. Anal. Math. 44 (194/1985), 1 - 20.

[12] Carbone, L. and S. Salerno: Homogenization with unbounded constraints on the gradient. Nonlin. Anal. 9 (1985), $431-444$.

[13] Ciarlet, P. G.: The Finite Element Method for Elliptic Problems. Amsterdam et al.: North-Holland Publ. Comp. 1978.

[14] Corbo Esposito, A. and R. De Arcangelis: A characterization of families of function sets described by constraints on the gradient. Ann. Inst. H. Poincaré Anal. Non Linéaire 11 (1994), 553 - 609.

[15] Corbo Esposito, A. and R. De Arcangelis: Homogenization of Dirichlet problems with nonnegative bounded constraints on the gradient. J. d'Anal. Math. 64 (1994), $53-96$. 
[16] Corbo Esposito, A. and R. De Arcangelis: A characterization of sets of functions and distributions on $\mathbb{R}^{n}$ described by constraints on the gradient. J. Convex Anal. 3 (1996), $167-194$.

[17] Dal Maso, G.: An Introduction to $\Gamma$-Convergence (Progress in Nonlinear Differential Equations and their Applications: Vol. 8). Basel: Birkhäuser 1993.

[18] De Maio, U. and T. Durante: Homogenization of Dirichlet problems for some types of integral functionals. Ric. Mat. 46 (1997), $177-202$.

[19] Garroni, A., Nesi, V. and M. Ponsiglione: Dielectric Breakdown: optimal bounds. R. Soc. Lond. Proc., Ser. A: Math. Phys. Eng. Sci. 457 (2001), 2317 -2335 .

[20] Glowinski, R. and H. Lanchon: Torsion élastoplastique d'une barre. J. de Méc. 12 (1973), $151-171$.

[21] Kohn, R. V. and T. D. Little: Some model problems of polycrystal plasticity with deficient basic crystals. SIAM J. Appl. Math. 59 (1998), 172 - 197.

Received 12.07.2002 